\title{
ELECTRON-INDUCED SURFACE CHEMISTRY ON TIN IN ULTRAHIGH VACUUM*
}

\author{
Qing $\mathrm{Ma}^{\dagger}$ and Richard A. Rosenberg, Argonne National Laboratory, Argonne, IL 60439
}

\begin{abstract}
The property of the TiN/vacuum interface in ultrahigh vacuum and under electron beam irradiation is studied using Auger electron spectroscopy. About one monolayer of oxygen adsorbs on the surface and metal oxidation occurs. The characteristic of oxygen adsorption is discussed. Under electron irradiation with energies ranging from $1 \mathrm{keV}$ to $9 \mathrm{keV}$, carbon accumulates on the surface, in addition to oxygen adsorption, which modifies the surface chemistry to an extent that depends on the vacuum conditions, electron current density, and electron beam energy. High-energy electrons induce $\mathrm{TiC}$ formation.
\end{abstract}

\section{INTRODUCTION}

The secondary electron yield (SEY) of TiN films is low; therefore, it is the coating of choice for accelerator components to reduce secondary electron production. Secondary electrons can have a deleterious effect on beam stability due to electron cloud effect and on the performance of components such as storage ring rf tuners due to temperature rise [1]. The SEY characteristics of TiN have been studied previously [2]. Electron impact also induces chemical changes on surfaces through processes like surface species desorption and adsorbate dissociation or deposition. However, changes in the surface chemistry of TiN that may occur as a result of electron irradiation are little known. In particular, the exact chemical information of these surfaces is lacking. Since electrons in accelerators are ubiquitous, with energies ranging from $\mathrm{MeV}$ to a few $\mathrm{eV}$ (secondary electrons), understanding these changes on TiN surfaces in ultrahigh vacuum (UHV) ambient, is important.

We present an Auger electron spectroscopy (AES) study of the electron-induced surface chemistry on TiN films in UHV.

\section{EXPERIMENTAL}

The TiN films were deposited on $\mathrm{Si}$ wafers using a magnetron sputtering system and were loaded into a UHV system. Surface contaminants were removed by 20-min $\mathrm{Ar}^{+}$sputtering. The $\mathrm{Ar}^{+}$ion kinetic energy was set at 1.5 $\mathrm{keV}$ with a measured sample current of $\sim 1.5 \mu \mathrm{A}$. The ionincident angle was $60^{\circ}$ to the sample surface normal. The base pressure of the UHV system was $2.5 \times 10^{-10}$ Torr, while the pressure near the sample was $3 \times 10^{-9}$ Torr when the gate valve to the intro-chamber was open. The mass spectra measured at these pressures showed that residual gases were mainly $\mathrm{H}_{2}, \mathrm{H}_{2} \mathrm{O}, \mathrm{O}_{2}, \mathrm{CO}$ or $\mathrm{N}_{2}$, and $\mathrm{CO}_{2}$.

The electron gun operates up to $10 \mathrm{keV}$ with the spot size controllable by electrostatic lenses. Electrons with energies from 1-9 $\mathrm{keV}$ were used to irradiate the TiN surface. The e-beam current, $I_{e}$, was determined using a Faraday cup. Electron current densities for irradiation ranged from $3 \times 10^{-4}$ to $1.25 \times 10^{-3} \mathrm{~A} / \mathrm{cm}^{2}$. The low-currentdensity beam was used for the SEY measurements to minimize beam effect on the surface. The SEY was measured as a function of impact electron energies using a retarding potential method [2] equal to $\left(I-I_{s} / I_{e}\right)$. The sample current, $I_{s}$, was recorded by an electrometer. The AES was used to monitor the surface chemistry. The Auger electrons generated by the irradiation electrons were collected using a hemispheric energy analyzer.

\section{AES AND SEY OF TIN}

Figure 1 shows the AES spectra of various TiN surfaces: (a) as-received, (b) $\mathrm{Ar}^{+}$sputter-cleaned, and (c) the clean surface after $450 \mathrm{~min}$ at $3 \times 10^{-9}$ Torr. After ion sputtering, the residual oxygen in the TiN film was negligibly small. Using the method reported in [3], the composition of the TiN film was determined to have a $\sim 1: 1$ stoichiometry. After $450 \mathrm{~min}$ in UHV, oxygen, but no carbon, adsorbed on the surface (see Fig. 1c).

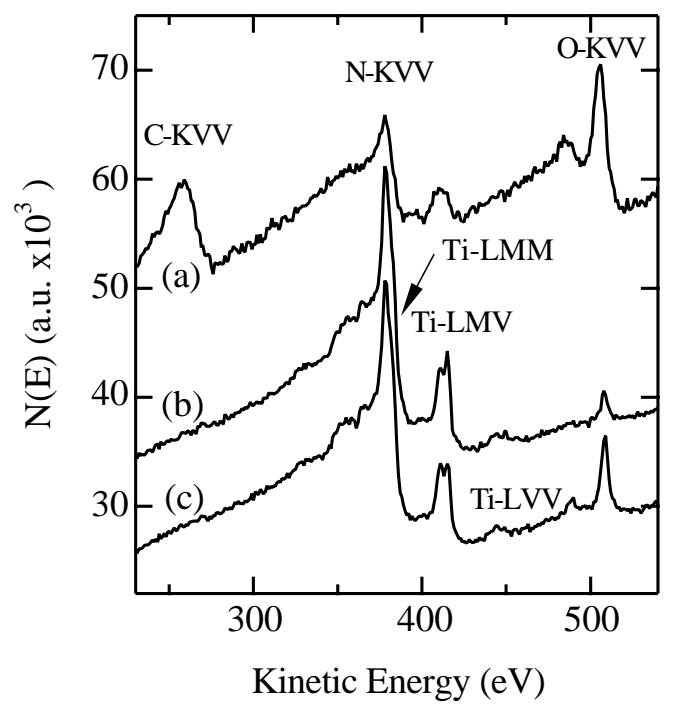

Figure 1: AES spectra of (a) as-received, (b) sputtercleaned, and (c) $450 \mathrm{~min}$ at $3 \times 10^{-9}$ Torr.

\footnotetext{
* The work is supported by the U.S. Department of Energy, Office of Basic Energy Sciences under contract No. W-31-109-ENG-38.

† qingma@aps.anl.gov
} 
Figure 2 shows the SEYs measured on the three surfaces. For the clean surface, $\sigma_{\max } \sim 1$ (Fig. 2b). Note that the difference is rather small between it and the oxidized surface in vacuum after $450 \mathrm{~min}$. This slight increase in the SEY following oxidation is possibly due to reduction of electron scattering near the Fermi level, where the density of states decreases as a result of the transition of a metallic surface into a more insulating one. The SEYs of the TiN films in Fig. 2 correlate well with previously reported data $[2,4]$.

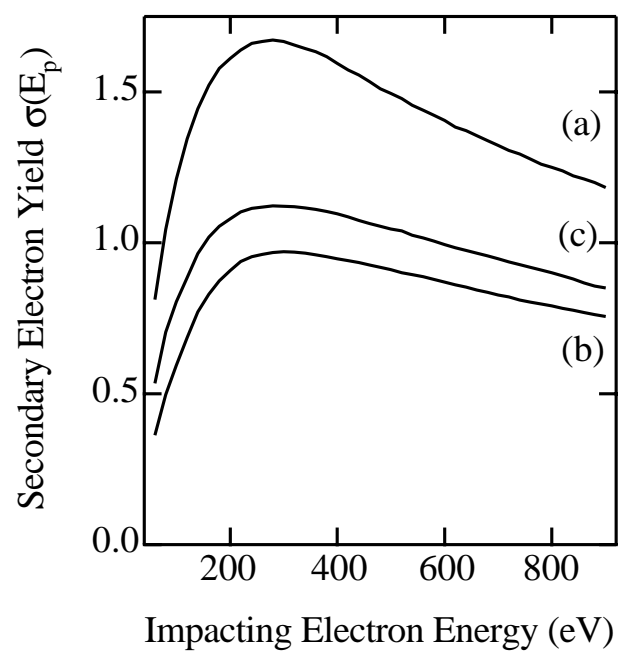

Figure 2: SEY vs $\mathrm{E}_{\mathrm{p}}$ for (a) as-received, (b) cleaned, and (c) oxidized in vacuum (see Fig. 1).

Band structure studies of TiN show considerable metalligand mixing in the density of states near the Fermi level, i.e., a significant contribution of states with $d$ symmetry to the N 2p states and vice-versa [5]. Intrabond transitions originating from creation of $\mathrm{L}$ core holes on $\mathrm{Ti}$ are thus possible, resulting in two branches in the TiLMV line (Fig. 1). The branch at higher kinetic energy is due to the direct transitions, and the one at lower kinetic energy is due to the intrabond transitions. The latter is sensitive to changes in the valence band upon the chemical interaction of TiN with adsorbates. Its intensity thus increases with oxidation (see Fig. $1 b$ and c). For TiN, the Ti-LMM line mixes with the N-KVV line.

\section{OXIDATION CHARACTERISTICS}

As shown in Fig. 1, the TiN surface oxidizes in a vacuum of $3 \times 10^{-9}$ Torr (and also at $2.5 \times 10^{-10}$ Torr). Oxidation is clearly indicated by enhancement of the intrabond transitions and relative decrease of the direct transitions, of the Ti-LMV line. By measuring the AES spectra as a function of time, the oxidation process in UHV is monitored. Figure 3 plots Auger peak heights vs time for a surface oxidized at $3 \times 10^{-9}$ Torr. The initial oxygen adsorption is rapid, then slows down dramatically and virtually stops after some time. The Ti-LMM intensity, as well as that of N-KVV (not shown), attenuates with time accordingly. There is no evidence of carbon accumulation on the surface for this time scale (see Fig. 1c). Assuming a uniform growth, the thickness, $h$, of the adsorbed layer is estimated to be one monolayer (ml) at the saturation point, $h=\lambda \ln \left(I_{d} I_{\text {sub }}\right)$, where $I_{o}$ and $I_{s u b}$ are the substrate signals before and after adsorption, and $\lambda=10.6 \AA$, the mean free path for the $384-\mathrm{eV} \mathrm{Ti}$ LMM Auger electrons.

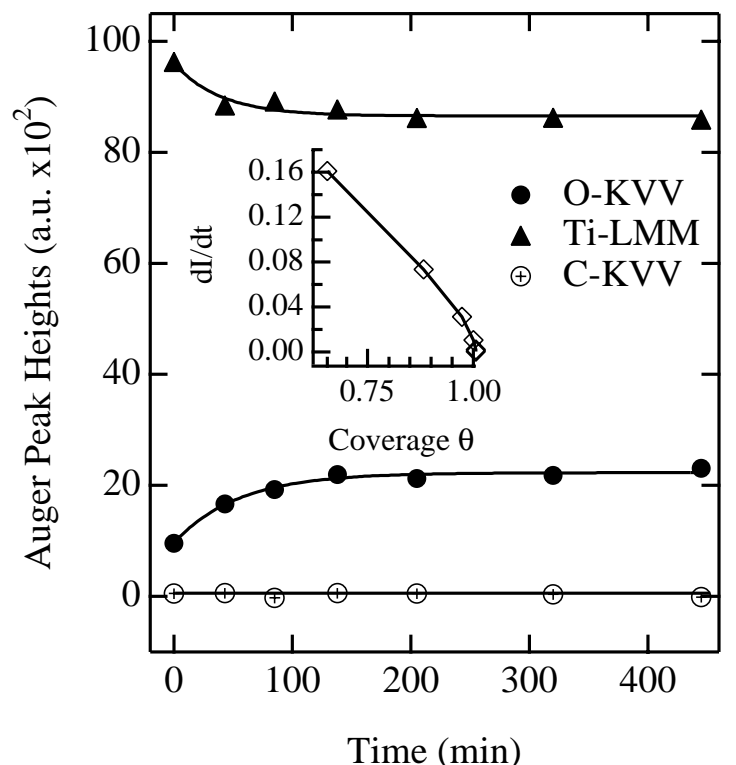

Figure 3: Auger peak heights of O-KVV, Ti-LMM, and $\mathrm{C}-\mathrm{KVV}$ vs time $\left(3 \times 10^{-9}\right.$ Torr). The full lines are the simulations. Insert: $\mathrm{O}$ growth rate vs coverage.

The nonlinear behavior of the oxygen growth kinetics does not fit the layer-by-layer growth mode in which the Auger intensities vary linearly with coverage $\theta$ within the growth of each $\mathrm{ml}$ [6]. However, it fits an exponential function, which implies a Volmer-Webber island growth. In this growth mode, the island height may be estimated to be $25 \AA$ with only a $10 \%$ coverage, which suggests an unlikely, if not impossible, film morphology.

The oxygen growth may reflect the $\theta$-dependence of the sticking coefficient, $s$, within the first ML regime, which varies nonlinearly for $\theta>0.5$ [7]. $s$ is defined as the condensation rate, $\kappa$, over the gas impingement rate, $v$ $\left(\mathrm{cm}^{-2} \mathrm{sec}^{-1}\right)$, i.e., $s=\kappa / v . \kappa$ may be assumed to be proportional to the oxygen growth rate, $\eta(=d I / d t)$. Since $v$ is constant for a given pressure, $s$ is proportional to $\eta$. The inset in Fig. 3 plots $\eta$ vs $\theta$. The values are obtained for a uniform layer growth and assume a surface density of $10^{15}$ atoms $\mathrm{cm}^{-2}$. This analysis may have limited accuracy; however, it does qualitatively describe the nonlinear behavior of the initial oxygen growth. If $\mathrm{H}_{2} \mathrm{O}$ is the main source of oxygen, for which $\mathrm{P}_{\mathrm{H}_{2} \mathrm{O}} \sim 2 \times 10^{-9}$ Torr, $\sim 11$ Langmuir $\mathrm{H}_{2} \mathrm{O}$ would be adsorbed on the surface at 100 min (1 Langmuir $=10^{-6}$ Torr-sec). This leads to an average $s \sim 0.1$. 


\section{5 e-INDUCED SURFACE CHEMISTRY}

Under 1- to 9-keV electron irradiation, the TiN surface accumulated carbon in addition to oxygen. Figure 4 compared a sputter-cleaned surface with the same surface in a vacuum of $3 \times 10^{-9}$ Torr after $485-\mathrm{min}, 9-\mathrm{keV}$ electron irradiation with an electron current density of $7.0 \times 10^{-4}$ $\mathrm{A} / \mathrm{cm}^{2}$. A prominent carbon peak appeared at $E_{k}=271 \mathrm{eV}$, and the oxygen peak increased as well. Carbon increased linearly with e-beam exposure (inset), and oxygen increased (not shown) with kinetics similar to that presented in Fig. 3 but with a slightly lower magnitude, (when comparing to the e-beam-irradiated surface with the surface oxidized in vacuum [Fig. 1c] for the same time scale). The details of Ti-LMV indicate that the intrabondtransition intensity increased only slightly, showing a weak metal oxidation despite the oxygen adsorption.

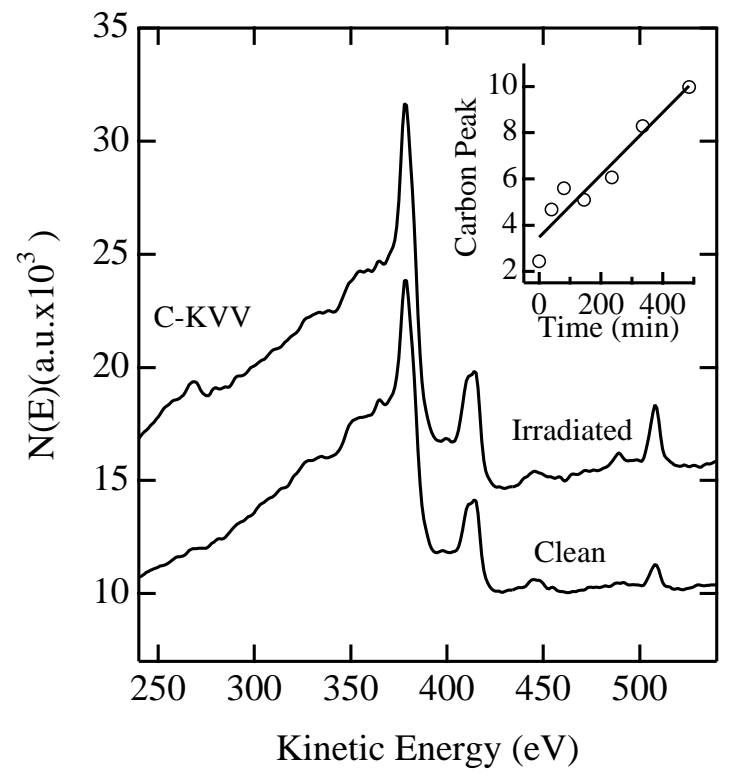

Figure 4: A clean surface irradiated for 485 min by $9-$ $\mathrm{kV}$ electrons. Inset: C-KVV vs time.

The surface chemistry of the electron-irradiated surface was studied by careful measurements of C-KVV vs exposure time. Carbon began to accumulate at the onset of the exposure. At $\sim 235 \mathrm{~min}$, prominent features related to titanium carbide appeared. The carbon deposition may have interrupted metal oxidation by forming metal carbide, a process that may be facilitated by the presence of oxygen [8]. Some of the oxygen adsorption may have occurred on the carbide surface, where oxygen is presumably channeled to bond to carbon. This accounted for the observed weak metal oxidation. For longer exposure times, a graphitic carbon feature appeared. For graphite $\sigma_{\max } \sim 1$. TiC also has a low SEY [2]. Unlike the UHV-oxidized surface (Fig. 1c) of which the SEY increased with oxidation (Fig. 2c and b), the SEY indicated no changes after 210-min, 9$\mathrm{keV}$ e-beam irradiation despite oxygen adsorption. The buildup of graphitic carbon and carbide on the surface may have caused this result. One may argue that the e-beaminduced damage in the oxide may also play a role [2].

For lower electron current densities smaller amounts of carbon and carbide were observed on the surfaces, with a consequent increase in metal oxidation. For lower electron energies carbon also adsorbed on the surface. But, metal oxidation dominated over carbide formation. The Ti $K$ shell ionization cross-sections for electron impact showed a maximum at $\sim 10 \mathrm{keV}$, which is five times larger than that near $5 \mathrm{keV}$ [9]. Thus, for $9-\mathrm{keV}$ electrons, the Ti $K$ shell excitation played a role in the $\mathrm{TiC}$ formation.

The mechanism of the carbon deposition may be similar to a process suggested in [10], in which carbon-containing gas molecules first adsorb reversibly on the surface and are then decomposed into carbon and other species by the incoming electrons, resulting in deposition. However, the effect of the secondary electrons on the process may also be significant, which will be the subject of further study.

\section{CONCLUSIONS}

In UHV the TiN surface is modified primarily through metal oxidation. About $1 \mathrm{ml}$ oxygen grows on the surface. The growth kinetic is determined by the $s-\theta$ relationship. Electron irradiation induces carbon deposition on the surface, which modifies the surface chemistry to an extent that depends on the vacuum conditions, electron current density, and electron beam energy. For high-energy electron beams, electrons induce $\mathrm{TiC}$ formation. For longer electron irradiation times the topmost carbon layer showed a distinct graphitic nature. For these surfaces, the SEYs is not very different from that of TiN.

\section{ACKNOWLEDGMENT}

We would like to thank Dean R. Walters for providing the TiN samples.

\section{REFERENCES}

[1] E.W. Hoyt and W. P. Schultz, SLAC Technical Note 75 (March 1975).

[2] E.L. Garwin et al., J. Appl. Phys. 61, 1145 (1987).

[3] J.E. Sundgren, A. Rockett, and J.E. Greene, J. Vac. Sci. Technol. A 4, 2770 (1986).

[4] P. Prieto and R.E. Kirby, J. Vac. Sci. Technol. A 14, 2819 (1995).

[5] H. Hochst et al., Phys. Rev. B 25, 7183 (1982).

[6] C. Argile and G.E. Rhead, Surf. Sci. Rep. 10, 277 (1989).

[7] P. A. RedHead et al., The Physical Basis of Ultrahigh Vacuum, AIP, New York (1993).

[8] J. Long and K.W. Sykes, Proc. Phys. Soc. London Sect. A 215, 100 (1952).

[9] M. Liu et al., Atomic Data and Nuclear Data Tables 76, 213 (2000).

[10] A.J.M. Mens and O.L.J. Gijzeman, Appl. Surf. Sci. 99, 133 (1996). 